\title{
Tratamento antitabágico a base de reposição de nicotina em mais de um ano de acompanhamento: uma revisão sistemática
}

\author{
Anti-smoke treatment based on nicotinical replacement in more than one year of follow-up: \\ a systematic review
Tratamiento antihumo basado en el reemplazo de nicotina en más de un año de seguimiento: una revisión sistemática

Julia Aires Thomaz Maya ${ }^{1 *}$, Frederico Oásis Oliveira ${ }^{1}$, Roberto José Bittencourt ${ }^{1}$.

\section{RESUMO}

Objetivos: Analisar o sucesso do tratamento farmacológico com foco na reposição de nicotina para cessação de tabagismo em pacientes com acompanhamento por no mínimo 1 ano após instituição terapêutica. Métodos: Tratase de uma revisão sistemática conforme protocolo PRISMA. Inicialmente foram levantados 748 artigos, após seleção pela leitura de resumos 5 artigos foram submetidos aos instrumentos "Cochrane risk of Bias" e da qualidade dos artigos pelo sistema GRADE de evidência de qualidade. Resultados: Os artigos selecionados representavam ensaios clínicos randomizados que utilizaram reposição de nicotina para cessação do tabagismo, a análise dos ensaios permitiu concluir que não há diferença estatística na efetividade do tratamento entre as diferentes formas de reposição de nicotina quando comparadas ao tratamento tradicional já bem estabelecido em literatura, levando em conta as taxas de abstinência após 1 ano da cessação do uso de tabaco. Considerações finais: A reposição de nicotina é eficaz na cessação do tabagismo após 1 ano de tratamento independentemente do método de reposição.

Palavras-chave: Abandono do uso de tabaco, Abandono do hábito de fumar, Tabagismo, Agentes de cessação do hábito de fumar, Resultado de tratamento.

\begin{abstract}
Objectives: To analyze the success of pharmacological treatment with a focus on the formation of nicotine for smoking cessation in patients followed up for at least 1 year after therapy. Methods: This is a literature review according to the PRISMA protocol. With the initial selection of 748 articles, 5 articles were selected, after final screening, which were evaluated using the "Cochrane risk of Bias" and using the GRADE quality evidence system to measure the quality of the articles. Results: The selected articles represent the randomized clinical trials that use the result of nicotine for smoking cessation, an analysis of the trials, that there is no statistical difference in the effectiveness of the treatment between the different forms of nicotine when compared to the traditional treatment already well established in the literature, taking into account the abstinence rates after 1 year of smoking cessation. Final considerations: Nicotine conversion is effective in smoking cessation after 1 year of treatment regardless of the partnership method.
\end{abstract}

Keywords: Tobacco use cessation, Smoking cessation, Tobacco use disorder, Smoking cessation agents, Treatment outcome.

\section{RESUMEN}

Objetivos: Analizar el éxito del tratamiento farmacológico con enfoque en el reemplazo de nicotina para el abandono del hábito de fumar en pacientes con seguimiento de al menos 1 año después de la institución terapéutica. Métodos: Se trata de una revisión de la literatura según el protocolo PRISMA. Inicialmente se recolectaron 748 artículos, luego de la selección mediante lectura de resúmenes, 5 artículos fueron sometidos al "Cochrane risk of Bias" y la calidad de los artículos por el sistema de evidencia de calidad GRADE. Resultados: Los artículos seleccionados representaron ensayos clínicos aleatorizados que utilizaron reemplazo de nicotina para dejar de fumar, el análisis de los ensayos permitió concluir que no existe diferencia estadística en la efectividad del tratamiento entre las diferentes formas de reemplazo de nicotina en comparación con el tratamiento tradicional ya bien establecido. en la literatura, teniendo en cuenta las tasas de abstinencia después de 1 año de dejar de fumar. Consideraciones finales: El reemplazo de nicotina es eficaz para dejar de fumar después de 1 año de tratamiento, independientemente del método de reemplazo.

Palabras clave: Cese del uso de tabaco, Cese del hábito de fumar, Tabaquismo, Agentes para el cese del hábito de fumar, Resultado del tratamiento.

1 Escola Superior de Ciências da Saúde (ESCS), Brasília - DF. *E-mail: juliaairesthomazmaya@gmail.com 


\section{INTRODUÇÃO}

O tabagismo é a principal causa de doenças preveníveis e óbitos evitáveis em todo o mundo (GIFFORD EV, et al., 2011). A Organização Mundial de Saúde (OMS) preconiza a integração da vigilância dos programas de tabaco a nível nacional, regional e global para que as consequências do uso de tabaco sejam minimizadas. Estima-se que em $201520,2 \%$ da população mundial com idade maior ou igual a 15 anos era fumante, representando redução de $6,7 \%$ em relação ao ano 2000 e de $4,1 \%$ em relação a 2005 . Assim, estabeleceuse o objetivo de redução das taxas mundiais de tabaco para $17.3 \%$ entre a população com mais de 15 anos até 2025 , sendo para a América a redução projetada para $13 \%$ da população no mesmo ano (WORLD HEALTH ORGANIZATION, 2018).

No Brasil, os dados mais recentes sobre o tema são do DATASUS, de 2013, em que $15 \%$ da população brasileira era usuária de tabaco. Ademais, populações com maior de vulnerabilidade social têm maiores taxas de tabagismo (RICHMOND R, et al., 2013). Os dados demonstram que 51,1\% dos fumantes tentaram parar de fumar nos últimos 12 meses e que cerca de $8 \%$ deles procurou tratamento para parar de fumar (DATASUS, 2019). A relevância da cessação do tabagismo se apoia no fato de que tabaco está implicado como fator de risco em mais de 50 doenças, entre essas doenças crônicas, como: hipertensão arterial, doenças coronarianas, câncer de pulmão, faringe, esôfago e estômago. Além de estar implicado em pior qualidade de vida em relação aos não dependentes de tabaco.

Dessa forma, a cessação do uso dessa droga minimiza a chances de ocorrência de comorbidades associadas, futuras hospitalizações, óbitos evitáveis e melhora a qualidade de vida do indivíduo, bem como reduz gastos em saúde (CARE P, 2000; BARTECCHI CE, et al., 1994; CASTRO MG, et al., 2007). A nicotina, princípio ativo do tabaco, apresenta ação no sistema dopaminérgico mesocorticolímbico mediada pela ligação a receptores colinérgicos nicotínicos, consequentemente, promove aumento da dopamina nesse circuito, também medeia alterações moleculares que levam a dependência da droga em usos prolongados (PLANETA CS e CRUZ FC, 2005). Em função de gerar dependência pacientes podem apresentar síndrome de abstinência com retirada da droga.

A longo prazo, essas mesmas alterações no sistema mesocorticolímbico atuam no fator motivacional de fumar, o que favorece recaídas. Nesse sentido, maiores taxas de sucesso na abstinência podem ser obtidas se os tratamentos antitabágicos farmacológicos forem utilizados (FRANÇA SAS, et al., 2015). As taxas de sucesso na abstinência do tratamento farmacológico antitabágicos variam de acordo com a medicação utilizada e entre combinações dessas medicações. Estudos publicados pela Cochrane já estabeleceram as taxas de abstinência em pacientes com seguimento pós tratamento por no mínimo 6 meses (LINDSON N, et al., 2019; CAHILL K, et al., 2013).

Entretanto não há estudos de revisão na literatura ou metanálises que analisem a cessação do tabagismo com 1 ano ou mais de segmento. Portanto, este trabalho visa analisar o sucesso dos tratamentos farmacológicos que empregam a reposição de nicotina para cessação de tabagismo em pacientes com acompanhamento por no mínimo 1 ano após instituição da terapia antitabágica.

\section{MÉTODOS}

Realizou-se uma revisão sistemática nas bases de dados PubMed®, Literatura Latino-Americana e do Caribe em Ciências da Saúde (LILACS), Embase®, Índice Bibliográfico Español en Ciencias de la Salud (IBECS), Scientific Electronic Library Online (SCIELO), Cochrane Central Register of Controlled Trials (CENTRAL) e Journal of the American Medical Association (JAMA) conforme protocolo Preferred Reporting Items for Systematic reviews and Meta-Analyses (PRISMA) e Manual do Ministério da Saúde sobre Revisão Sistemática com Metanálise. A busca de artigos utilizou os Descritores em Ciências da Saúde (DeCs) combinados com operadores booleanos: "tobacco use cessation products" AND "Tobacco use cessation" AND "treatment outcome".

Para seleção primária de artigos não houve limitação de idioma. Inicialmente foram identificados 748 artigos publicados nos últimos 10 anos. Desses, 5 na base de dados da Scielo, 254 na PUBMED, 175 no JAMA, 139 na CENTRAL, 12 na IBECS, 4 na LILACS e 159 na EMBASE. A última busca foi realizada em 
novembro de 2019. Após o resultado inicial das buscas, esses artigos foram submetidos a uma nova seleção baseada na leitura da metodologia sendo então selecionados apenas artigos que tiveram seguimento dos pacientes por 1 ano ou mais.

Assim foram selecionados 85 artigos com acompanhamento de pacientes por 1 ano ou mais com terapêutica antitabágica farmacológica e não farmacológica. Após a exclusão de artigos duplicados restaram 77 artigos com acompanhamento por no mínimo 1 ano após instituição de algum tipo de terapia antitabágica (Figura 1).

No intuito de realizar uma metanálise consistente para definição do estado da arte do tratamento farmacológico atual com seguimento dos pacientes por mais de 1 ano publicados após overwiew da Cochrane, pois esta tinha como foco trabalhos com seis meses de seguimento, os artigos passaram por uma nova seleção.

Dois avaliadores independentes selecionaram, a partir da leitura completa dos artigos, ensaios clínicos randomizados, que realizaram intervenção farmacológica, com populações livres de doenças psiquiátricas, não gestantes, na faixa etária de 10 à 80 anos que tenham sido publicados à partir de 2013. Sendo então selecionados 5 artigos para esse estudo, todos eles avaliando reposição de nicotina para cessação do tabagismo. Devido a divergência de protocolos terapêuticos instituídos nos estudos a metanálise foi inviabilizada.

Figura 1 - Diagrama da metodologia da seleção de artigos.

BUSCA PRIMÁRIA COM OPERADORES BOOLEANOS: 748 ARTIGOS

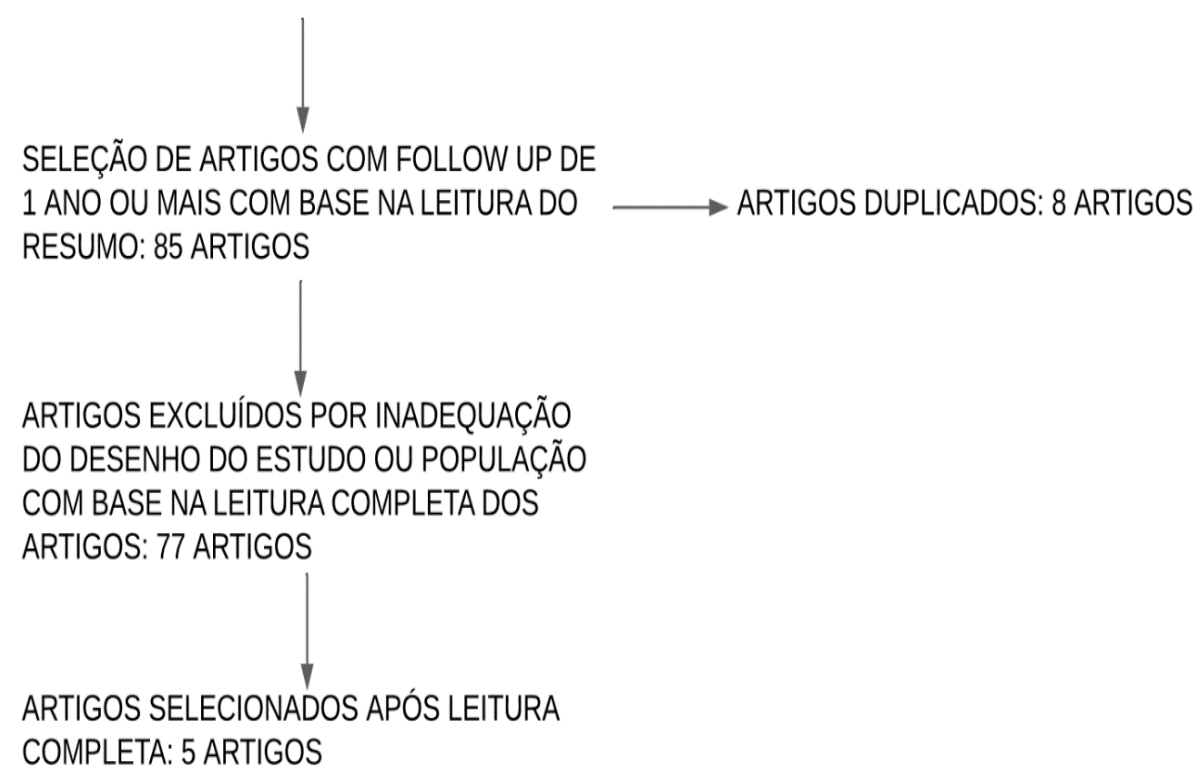

Fonte: Maya JAT, et al., 2020.

A fim de verificar a confiabilidade dos resultados aqui apresentados os artigos selecionados foram analisados por meio do instrumento "Cochrane risk of Bias". Avaliou-se o risco de vieses de alocação, sigilo de alocação, mascaramento de participantes e equipe, mascaramento na avaliação dos desfechos, apresentação de dados incompletos de desfecho, relato seletivo de desfecho ou outros vieses que os estudos selecionados possam apresentar (AVEYARD P, 2018; NELSON PR, et al., 2019).

Em relação tanto ao mascaramento de participantes e equipe quanto ao mascaramento na avaliação do desfecho sobre as técnicas de cessação do tabagismo verificou-se que não foi possível sua realização em nenhum dos estudos pela diferença das técnicas farmacológicas utilizadas, muitas delas requeriam que 0 paciente tivesse consciência do tratamento que estava utilizando para fazer uso adequado da terapêutica. Em todos os estudos selecionados a randomização e o sigilo de alocação dos pacientes foi adequado. A síntese dos riscos de vieses dos artigos em respectivas categorias está apresentada na (Quadro 1). 
Quadro 1 - Cochrane risk of Bias Tool. Avaliação do risco de viés.

\begin{tabular}{|c|c|c|c|c|c|c|c|}
\hline Artigo & Randomização & $\begin{array}{c}\text { Sigilo de } \\
\text { Alocação }\end{array}$ & $\begin{array}{c}\text { Mascaramento } \\
\text { de } \\
\text { participantes e } \\
\text { equipe }\end{array}$ & $\begin{array}{c}\text { Mascaramento } \\
\text { na avaliação } \\
\text { do desfecho }\end{array}$ & $\begin{array}{c}\text { Dados } \\
\text { incompletos } \\
\text { de desfecho }\end{array}$ & $\begin{array}{c}\text { Relato } \\
\text { seletivo } \\
\text { de } \\
\text { desfecho }\end{array}$ & $\begin{array}{c}\text { Outros } \\
\text { vieses }\end{array}$ \\
\hline $\begin{array}{c}\text { Hajek P, } \\
\text { et. } \\
\text { al. (2019) }\end{array}$ & Baixo & Baixo & Alto & Alto & Baixo & Baixo & Baixo \\
\hline $\begin{array}{c}\text { Baker TB, } \\
\text { et al. } \\
(2016)\end{array}$ & Baixo & Baixo & Alto & Moderado & Baixo & Baixo & Baixo \\
\hline $\begin{array}{c}\text { Aveyard } \\
\text { P, 2018 }\end{array}$ & Baixo & Baixo & Alto & Moderado ${ }^{1}$ & Moderado & Moderado & Baixo \\
\hline $\begin{array}{c}\text { Schnoll } \\
\text { RA et al. } \\
(2015)\end{array}$ & Baixo & Baixo & Alto & Moderado & Baixo & Baixo & Moderado3 \\
\hline $\begin{array}{c}\text { Nelson } \\
\text { PR, et al. } \\
(2019)\end{array}$ & Baixo & Baixo & Alto ${ }^{1}$ & Moderado & Baixo & Baixo & Baixo \\
\hline
\end{tabular}

Legenda: ${ }^{1}$ Os estudos não conseguem realizar o mascaramento na avaliação do desfecho nem o cegamento pois os pacientes precisam comprar parte da medicação para repor;

${ }^{2} \mathrm{O}$ estudo não descreve quais são as terapias medicamentosas possivelmente usadas pelo grupo controle; ${ }_{3} \mathrm{O}$ estudo considera como lapso ou recaída o ato de fumar por 7 dias consecutivos, em divergência com ensaios clínicos anteriormente publicados.

Fonte: Maya JAT, et al., 2020.

Os artigos também foram avaliados pelo sistema GRADE de evidência de qualidade, Hajek $P$, et. al. (2019) e Baker TB, et al. (2016) apresentaram alta qualidade de evidência pelo sistema GRADE, os demais trabalhos apresentaram evidência moderada.

\section{RESULTADOS}

No trabalho de Hajek $P$, et al. (2019) foi realizada intervenção com cigarro eletrônico, fornecimento de cigarro eletrônico recarregável com líquido contendo uma concentração de $18 \mathrm{mg} / \mathrm{ml}$ de nicotina. Comparouse essa intervenção a diferentes tipos de reposição nicotínica, como nicotina com adesivos, goma de mascar, inalantes de nicotina, spray nasal, bem como combinações de diferentes tipos de reposição. Foram randomizados 886 pacientes, destes 356 do braço da intervenção com cigarro eletrônico e 342 do braço de reposição nicotínica por meio das outras técnicas de reposição já descrita completaram o estudo e ambos foram avaliados após 1 ano.

A taxa de abstinência sustentada em 1 ano foi de $18.0 \%$ para cigarro eletrônico e $9.9 \%$ para o grupo de reposição de nicotina por meio de outras técnicas (Risco Relativo [RR], 1.83; 95\% intervalo de confiança [IC], 1.30-2.58; $P$ <0.001). Havendo um melhor resultado na reposição nicotínica com cigarro eletrônico quando comparada a outras formas de reposição da nicotina. Ressalta-se que em 1 ano de acompanhamento $80 \%$ dos pacientes ainda fazia uso do cigarro eletrônico e $9 \%$ ainda fazia uso da reposição de nicotina por meio de outras técnicas.

Baker TB, et al. (2016) realizaram intervenção farmacológica por 12 semanas por meio de três esquemas terapêuticos: uso de terapia de reposição de nicotina combinada (TNC) por meio de adesivo de nicotina associado a reposição de nicotina oral e comparada ao uso de Vareniclina associado a adesivo de nicotina e ao uso de reposição nicotínica apenas com adesivo. Ainda nesse estudo, pacientes alocados no braço de tratamento com vareniclina receberam uma carga do medicamento antes do dia de cessação do tabagismo como adaptação à dosagem terapêutica, gradativa do medicamento até a dose de $1 \mathrm{mg}$, quando era realizado abandono do tabaco, a partir de então permaneceram com a dose de $1 \mathrm{mg}$ por dia duas vezes ao dia por 11 semanas, sendo a dose do fármaco modificada de acordo com o perfil de efeitos colaterais da droga (BAKER TB, et al., 2016). 
A reposição de nicotina com adesivo, tanto no grupo de reposição combinada com vareniclina, quanto no grupo de reposição apenas de nicotina, foi realizada da mesma forma, sendo $21 \mathrm{mg}$ ao dia de nicotina via transdérmica por 8 semanas, com redução gradual da dose pelas 4 semanas seguintes e iniciada no dia da cessação do tabaco.

Além da terapia transdérmica pacientes randomizados no braço de terapia combinada receberam pastilhas de nicotina para uso oral de $2 \mathrm{mg}$ a $4 \mathrm{mg}$, e foram orientados a usar no mínimo 5 pastilhas ao dia por 12 semanas. Foram randomizados 1086 pacientes, 421 no braço da TNC, 424 no braço da vareniclina e 241 na terapia transdérmica isolada.

As taxas de abstinência ao final de 1 ano de segmento foram de $20.8 \%$ no grupo que utilizou adesivo transdérmico, $19.1 \%$ no grupo de reposição de vareniclina e de $20.2 \%$ no grupo que utilizou a terapia de reposição de nicotina associada. Comparando a reposição entre vareniclina $X$ TNC, não houve diferença significativa no resultado do tratamento em acompanhamento de 1 ano (RR -1.1 (-6.4 to 4.3);p 0.69) o mesmo ocorreu com a terapia de adesivo transdérmico X TNC (RR 0.56 (-5.8 to 7.0); p 0.86) (BAKER TB, et al., 2016).

Aveyard P (2018) estudou a eficácia da realização de uma pré-carga de nicotina, que deveria ser feita antes de o tratamento antitabastico de escolha se instituído, independente de qual fosse (bupropiona, vareniclina, adesivos transdérmicos, goma de nicotina, pastilhas de nicotina e associação entre esses).

Pacientes randomizados no braço da pré-carga de nicotina realizaram reposição com adesivos de nicotina de $21 \mathrm{mg} / 24 \mathrm{~h}$ por 4 semanas, a partir da data de cessação do tabagismo. Foram randomizados inicialmente 1792 pacientes, sendo que para validação final de 12 meses de acompanhamento participaram 204 no grupo que havia feito a pré-carga de nicotina e 171 pacientes no grupo controle.

Do grupo que recebeu a pré carga nicotínica 14.0\% conseguiu manter a abstinência em 12 meses de acompanhamento, já no grupo que não realizou a pré carga de nicotina 11,3\% mantiveram-se abstêmios. Comparando-se ambos tratamentos não houve diferença significativa entre eles (RR 1.24 (0.97 - 1.58); $p$ 0.09).

Contudo, o trabalho demonstrou que havia melhores resultados em todas as fases de avaliação da cessação do tabagismo quando a droga utilizada após o dia de cessação do tabagismo era a vareniclina. A avaliação de 12 meses também apresentou resultados significativos dessa associação (RR 1,30 $(1,02-1,66)$ IC 95\%, p 0,04). Um fator limitante para essa associação é o perfil de efeitos colaterais das drogas em associação, que por vezes fazem com que as doses utilizadas de vareniclina tenham que ser reduzidas. Schnoll RA, et al. (2015) realizaram três formas de intervenção terapêutica.

O primeiro grupo de pacientes (standart) recebeu adesivo transdérmico de nicotina $21 \mathrm{mg} / \mathrm{dia}$ por 8 semanas. O segundo (estendido) e terceiro grupo (manutenção) recebeu nicotina por 24 e 52 semanas, respectivamente, na mesma dose do primeiro grupo. Participaram do braço de tratamento standard 180 participantes, do estendido 163 participantes e do de manutenção 172 participantes.

No acompanhamento de 1 ano de segmento $23.8 \%$ dos participantes do tratamento standard e do tratamento estendido estavam em abstinência, enquanto $20,3 \%$ dos pacientes que estavam no grupo de manutenção seguiam abstinentes. O modelo de análise multivariada demonstrou que não houve significância estatística para os benefícios do tratamento estendido em comparação com as outras formas de tratamento na avaliação de 1 ano de segmento (OR de 1.17 (95\% Cl, 0.69-1.98; $\mathrm{P}=.57)$ ). Nelson PR, et al. (2019) avaliou a reposição de tabaco úmido em pastilhas de $600 \mathrm{mg}$ colocadas entre a bochecha e a gengiva, que deveriam ser utilizadas quantas vezes os pacientes randomizados julgassem necessário versus pastilhas com $4 \mathrm{mg}$ de nicotina conforme a necessidade dos pacientes, não passando de 5 pastilhas a cada 6 horas.

Após 1 ano de acompanhamento, apenas $0,9 \%$ dos pacientes em reposição com pastilhas de nicotina e $1,4 \%$ dos pacientes em reposição com tabaco úmido mantiveram-se abstêmios, não apresentando significância estatística entre os grupos analisados. Em todos os estudos os pacientes foram orientados sobre a importância da cessação do tabagismo e receberam sessões de aconselhamento para o mesmo associado a intervenção farmacológica (Quadro 2).

REAS/EJCH | Vol.12(12) | e5200 | DOI: https://doi.org/10.25248/reas.e5200.2020 Página 5 de $\mathbf{9}$ 
Quadro 2 - Síntese dos resultados.

\begin{tabular}{|c|c|c|c|c|c|}
\hline Artigo & Intervenção & $\begin{array}{l}\text { Qualidade da } \\
\text { evidência } \\
\text { (GRADE) }\end{array}$ & Efeito relativo & Grupo controle & Validação da abstinência \\
\hline $\begin{array}{l}\text { Hajek P, et. al. } \\
\qquad(2019)\end{array}$ & $\begin{array}{l}\text { Fornecimento de cigarro } \\
\text { eletrônico recarregável com } \\
\text { líquido contendo nicotina com } \\
\qquad \begin{array}{c}18 \mathrm{mg} / \mathrm{ml} \\
\mathrm{N}=356\end{array}\end{array}$ & ALTA & $\begin{array}{c}\text { RR } 1.83 \\
(1.30-2.58) \\
p<0.001 \\
\text { IC } 95 \%\end{array}$ & $\begin{array}{l}\text { Reposição de nicotina sob diferentes } \\
\text { formas (goma de mascar, inalantes de } \\
\text { Nicotina, spray nasal, com incentivo a } \\
\text { combinações de diferentes tipos de } \\
\text { reposição nicotínica) } \\
\mathrm{N}=342\end{array}$ & $\begin{array}{l}\text { Teste do monóxido de } \\
\text { carbono expirado <8ppm e } \\
\text { ausência de autodeclaração } \\
\text { de retorno ao hábito de fumar }\end{array}$ \\
\hline $\begin{array}{l}\text { Baker TB, et al. } \\
\qquad(2016)\end{array}$ & $\begin{array}{l}\text { Uso de vareniclina } \\
\qquad \mathrm{N}=424\end{array}$ & ALTA & $\begin{array}{c}\text { RR }-1.1 \\
(-6.4 \text { to } 4.3) \\
\text { p } 0.69 \\
\text { IC } 95 \%\end{array}$ & $\begin{array}{l}\text { Uso de terapia de reposição de } \\
\text { nicotina transdérmica e de reposição } \\
\qquad \begin{array}{c}\text { oral } \\
\mathrm{N}=421\end{array}\end{array}$ & $\begin{array}{c}\text { Teste do monóxido de } \\
\text { carbono expirado }<8 \text { ppm e }<5 \\
\text { ppm, sendo realizadas duas } \\
\text { análises estatísticas, com } \\
\text { resultados similares }\end{array}$ \\
\hline $\begin{array}{l}\text { Baker TB, et al. } \\
\qquad(2016)\end{array}$ & $\begin{array}{l}\text { Reposição de nicotina em } \\
\text { adesivo } \\
\mathrm{N}=241\end{array}$ & ALTA & $\begin{array}{c}\text { RR } 0.56 \\
(-5.8 \text { to } 7.0) \\
\text { p } 0.86 \\
\text { IC } 95 \%\end{array}$ & $\begin{array}{l}\text { Uso de terapia de reposição de } \\
\text { nicotina transdérmica e de reposição } \\
\text { oral } \\
\mathrm{N}=421\end{array}$ & $\begin{array}{c}\text { Teste do monóxido de } \\
\text { carbono expirado }<8 \text { ppm e }< \\
5 \text { ppm, sendo realizadas duas } \\
\text { análises estatísticas, com } \\
\text { resultados similares }\end{array}$ \\
\hline $\begin{array}{l}\text { Aveyard P } \\
\text { (2018) }\end{array}$ & $\begin{array}{l}\text { Uso de pré-carga de nicotina com } \\
\text { adesivos de nicotina por } 4 \\
\text { semanas antes de alguma } \\
\text { intervenção terapêutica } \\
\qquad N=204\end{array}$ & MODERADA & $\begin{array}{c}\text { RR } 1.24 \\
(0.97-1.58) \\
\text { p } 0.09 \\
\text { IC } 95 \%\end{array}$ & $\begin{array}{c}\text { Grupo controle não realizou pré-carga } \\
\text { com nicotina antes da escolha de } \\
\text { algum método de cessação do } \\
\text { tabagismo } \\
\mathrm{N} 171\end{array}$ & $\begin{array}{l}\text { Teste do monóxido de } \\
\text { carbono expirado <10 ppm }\end{array}$ \\
\hline $\begin{array}{l}\text { Schnoll RA, et } \\
\text { al. (2015) }\end{array}$ & $\begin{array}{l}\text { Uso contínuo de adesivos de } \\
\text { nicotina, por } 52 \text { semanas } \mathrm{N}=172\end{array}$ & MODERADA & $\begin{array}{c}\text { OR } 1.17 \\
(0.69-1.98) \\
P=0.57 \\
\text { IC } 95 \%\end{array}$ & $\begin{array}{l}\text { Comparado ao tratamento estendido } \\
\text { (durante } 24 \text { semanas } / \mathrm{N}=173 \text { ) e ao de } \\
\text { tradicional ( } 8 \text { semanas } / \mathrm{N}=180 \text { ) }\end{array}$ & $\begin{array}{c}\text { Considerado abstêmio } \\
\text { pacientes com níveis de CO } \\
\text { expirado }<10 \text { ppm }\end{array}$ \\
\hline $\begin{array}{l}\text { Nelson PR, et } \\
\text { al. (2019) }\end{array}$ & $\begin{array}{l}\text { Reposição de nicotina em } \\
\text { adesivos } 4 \mathrm{mg} \text { por adesivo } \\
\qquad \mathrm{N}=213\end{array}$ & MODERADA & $\begin{array}{c}0.9 \\
(0.0,2.2) \\
\text { p não informado } \\
\text { IC } 95 \%\end{array}$ & $\begin{array}{l}\text { Comparado com reposição de tabaco } \\
\text { em forma de pastilhas com 600mg } \\
\qquad N=218\end{array}$ & $\begin{array}{l}\text { Monóxido de carbono }(\mathrm{CO}) \\
\text { expirado com níveis } \leq 8 \text { partes } \\
\text { por milhão }(\mathrm{ppm})\end{array}$ \\
\hline
\end{tabular}

Fonte: Maya JAT, et al., 2020. 


\section{DISCUSSÃO}

Drogas antitabágicas podem ser classificadas em duas classes principais - nicotínicas e não nicotínicas. As drogas nicotínicas constituem a primeira linha de tratamento e tem como fármacos componentes a terapia de reposição de nicotina, bupropiona e a vareniclina. As drogas não nicotínicas são a segunda linha de tratamento e tem como componentes a nortriptilina e a clonidina (REICHERT J, 2008). Atualmente são disponibilizados pelo Ministério da Saúde para o tratamento de tabagismo pelo Sistema Único de Saúde (SUS) os seguintes medicamentos: Terapia de Reposição de Nicotina, através adesivo transdérmico, goma de mascar e pastilha, e o Cloridrato de Bupropiona (BRASIL, 2019).

Recentemente foi publicado um estudo norteador sobre as técnicas de cessação do tabagismo pela Revista Brasileira de Cardiologia para todos os países de língua portuguesa, nesse estudo são reforçadas as formas de cessação do tabagismo existentes e endossadas pelo Ministério da Saúde, entre elas que incluem a reposição de nicotina (OLIVEIRA GMM, et al., 2019). Ainda no momento atual, a pandemia deflagrada pelo novo coronavírus reacendeu a discussão sobre a importância da cessação do tabagismo, devido ao acometimento pulmonar da doença, levando a OMS a declarar uma nova iniciativa para a auxiliar 1,3 bilhões de pessoas a pararem de fumar durante esse período de pandemia (ONU NEWS, 2020).

Em Overview da Chrocrane foi demonstrado que a Terapia de reposição de nicotina (TRN) é superior ao placebo e responsável por incremento de $80 \%$ na quantidade de pessoas que obtiveram êxito na cessação do tabagismo em acompanhamento de 6 meses. A combinação de TRN também se mostrou mais efetiva que formulações isoladas para o mesmo período de acompanhamento (CAHILL K, et al., 2013; WADGAVE U e NAGESH L, 2016; AGARWAL A, et al., 2017).

Nesse estudo a síntese das diferentes formas de reposição de nicotina e demonstrou que não existe significância estatística entre a reposição com pré carga de nicotina, tratamento prolongado e combinação de diferentes formas de reposição de nicotina, quando comparadas ao tratamento tradicional já bem estabelecido em literatura, levando em conta as taxas de abstinência após 1 ano da cessação do uso de tabaco. $O$ trabalho de Hajek P, et al. (2019) demonstra, ao término de 1 ano, que o cigarro eletrônico é estatisticamente superior a terapêutica tradicional, entretanto $80 \%$ dos pacientes alocados na intervenção com cigarro eletrônico ainda faziam uso dele durante a análise de 1 ano, podendo-se questionar a veracidade dos resultados.

Em trabalhos previamente realizados utilizando o cigarro eletrônico demonstrou-se efetividade dessa forma de terapêutica, mas foi questionada a sua validade (LAM C e WEST A, 2015; EL DIB R, et al., 2017). Do mesmo modo, esse trabalho reafirma o questionamento se é real a eficácia dessa forma de cessação uma vez que se pode considerar que estes pacientes apenas substituíram a forma de uso de seu cigarro. Esse trabalho demonstra que é possível uma taxa de sucesso entre 3-21\% de abstinência após 1 ano da cessação do tabagismo utilizando tratamento com reposição de nicotina de forma combinada ou isoladamente.

Isso representa um novo marco importante para avaliação das técnicas de reposição de nicotina para cessação do tabagismo, uma vez que trabalhos anteriores de síntese de evidências analisaram apenas até 6 meses de acompanhamento dos pacientes e que ratificam os resultados demonstradas em trabalhos anteriores, que acompanharam pacientes por menos tempo (EL DIB R, et al., 2017). Uma nova metanálise da Cochrane, publicada em 2019 mostra que não há diferença significante estatisticamente entre a marcação de uma data definida para a cessação do tabagismo versus a simples decisão de parar de fumar (CAHILL K, et al., 2013).

No trabalho Nelson PR, et al. (2019) foi levantado durante a discussão, que caso fosse considerado o ponto de cessação do tabagismo semana depois do início do tratamento seria possível uma maior taxa final de cessação do tabagismo ao término de um ano, sendo este o trabalho que apresentou o menor percentual de cessação do tabagismo entre os analisados, $3 \%$. No entanto compreendemos que para a metodologia dos ensaios clínicos conduzidos a marcação de uma data para a cessação do tabagismo ainda tem relevância para obtenção de resultados finais relevantes.

Apesar de todos os artigos estudados relatarem a realização de aconselhamento quanto às implicações do tabagismo e benefícios da cessação do uso de tabaco, não houve análise objetiva do impacto desse aconselhamento em associação à terapia medicamentosa, mostrando uma lacuna de avaliação deste tipo de 
intervenção no absenteísmo a longo prazo, quando associado a terapia medicamentosa comprovadamente eficaz. Para confirmação da abstinência, todos os trabalhos avaliaram a cessação do tabagismo de duas formas, primeiramente pelo autorrelato dos pacientes que estavam sem fumar e posteriormente estes pacientes foram submetidos a uma análise quantitativa para confirmação do autorrelato por meio do teste expirado do monóxido de carbono.

O teste de monóxido de carbono expirado abaixo de $9 \mathrm{ppm}$ apresenta sensibilidade de $60 \%$ e especificidade de $97 \%$ para mensurar a abstinência (PERKINS KA, 2013). Assim falsos positivos para a cessação do tabagismo poderiam ter sido incluídos na análise foram mitigados pelo relato do uso de tabaco, não houve análise objetiva do impacto desse aconselhamento em associação à terapia medicamentosa, mostrando uma lacuna de avaliação deste tipo de intervenção no absenteísmo a longo prazo, quando associado a terapia medicamentosa comprovadamente eficaz.

Para confirmação da abstinência, todos os trabalhos avaliaram a cessação do tabagismo de duas formas, primeiramente pelo autorrelato dos pacientes que estavam sem fumar e posteriormente estes pacientes foram submetidos a uma análise quantitativa para confirmação do autorrelato por meio do teste expirado do monóxido de carbono. O teste de monóxido de carbono expirado abaixo de 9 ppm apresenta sensibilidade de $60 \%$ e especificidade de $97 \%$ para mensurar a abstinência (PERKINS KA, 2013). Assim falsos positivos para a cessação do tabagismo poderiam ter sido incluídos na análise foram mitigados pelo relato.

\section{CONSIDERAÇÕES FINAIS}

A reposição de nicotina é a forma mais relacionada com a abstinência por mais de 1 ano, porém não permite dizer que diferentes formas de intervenção terapêutica com nicotina sejam superiores umas às outras em resultados para o absenteísmo à longo prazo. A análise realizada dos artigos possibilita concluir que existe um déficit na produção de ensaios clínicos randomizados com acompanhamento dos pacientes por mais de um ano para avaliação da cessação do tabagismo. Pesquisas futuras são necessárias para se avaliar a eficácia e segurança ao incremento das taxas de cessação do tabagismo à longo prazo de associações terapêuticas entre reposição de nicotina e demais medicamentos disponíveis para o tratamento de dependência ao uso de tabaco.

\section{REFERÊNCIAS}

1. AGARWAL A, et al. Nicotine Replacement Therapy: An insight. Int J Oral Health Med Res 2017;4(4):76-79.

2. AVEYARD P. Effects on abstinence of nicotine patch treatment before quitting smoking: parallel, two arm, pragmatic randomised trial. bmj, 2018; 361: k2164.

3. BAKER TB, et al. Effects of nicotine patch vs varenicline vs combination nicotine replacement therapy on smoking cessation at 26 weeks: a randomized clinical trial. Jama, 2016; 315(4): 371-379.

4. BARTECCHI CE, et al. The human costs of tobacco use. New England Journal of Medicine, 1994; 330(13): 907-912.

5. BRASIL. Ministério da Saúde. Protocolos Clínicos e Diretrizes Terapêuticas: Dependência a Nicotina. Brasília: Ministério da Saúde; 2014.

6. CAHILL K, et al. Pharmacological interventions for smoking cessation: an overview and network meta-analysis. Cochrane database of systematic reviews, 2013; 5 .

7. CARE P. A clinical practice guideline for treating tobacco use and dependence. Jama, 2000; 283 3244-3254.

8. CASTRO MG, et al. Qualidade de vida e gravidade da dependência de tabaco. Archives of Clinical Psychiatry (São Paulo), 2007; 34(2): 61-67

9. MINISTÉRIO DA SAÚDE. DEPARTAMENTO DE INFORMÁTICA DO SUS - DATASUS. Informações de Saúde, Epidemiológicas e borbidade: de dados. Disponível em: http://tabnet.datasus.gov.br/cgi/deftohtm.exe?pns/pnspf.def .Acesso em: 20/04/19.

10. IB R, et al. Electronic nicotine delivery systems and/or electronic non-nicotine delivery systems for tobacco smoking cessation or reduction: a systematic review and meta-analysis. BMJ open, 2017; 7(2).

11. FRANÇA SAS, et al. Factors associated with smoking cessation. Revista de Saúde Pública, 2015; 49(10).

12. GIFFORD EV, et al. Does acceptance and relationship focused behavior therapy contribute to bupropion outcomes? A randomized controlled trial of functional analytic psychotherapy and acceptance and commitment therapy for smoking cessation. Behavior therapy, 2011; 42(4): 700-715.

13. HAJEK $P$, et al. A randomized trial of e-cigarettes versus nicotine-replacement therapy. New England Journal of Medicine, 2019; 380(7): 629-637.

14. LAM C e WEST A. Are electronic nicotine delivery systems an effective smoking cessation tool ?. Canadian journal of respiratory therapy: CJRT= Revue canadienne de la therapie respiratoire: RCTR, $2015 ; 51(4): 93$.

REAS/EJCH | Vol.12(12) | e5200 | DOI: https://doi.org/10.25248/reas.e5200.2020 Página 8 de 9 
15. LINDSON N, et al. Smoking reduction interventions for smoking cessation. Cochrane Database of Systematic Reviews, 2019; 9

16. NELSON PR, et al. Randomized trial to compare smoking cessation rates of snus, with and without smokeless tobacco health-related information, and a nicotine lozenge. Nicotine and Tobacco Research, 2019; 21(1): 88-94.

17. OLIVEIRA GMM, de et al. Recomendações para a Redução do Consumo de Tabaco nos Países de Língua Portuguesa-Posicionamento da Federação das Sociedades de Cardiologia de Língua Portuguesa. Arquivos Brasileiros de Cardiologia, 2019; 112(4): 477-486.

18. OMS quer ajudar 1,3 bilhão de pessoas a parar de fumar durante pandemia. ONU NEWS, 2020. Disponível em: https://news.un.org/pt/story/2020/07/1719811. Acesso em: 19/07/2020;

19. PERKINS KA, et al. Optimal carbon monoxide criteria to confirm 24 -hr smoking abstinence. Nicotine \& Tobacco Research, 2013; 15(5): 978-982.

20. PLANETA CS, CRUZ FC. Bases neurofisiológicas da dependência do tabaco. Archives of Clinical Psychiatry (São Paulo), 2005; 32(5): 251-258.

21. REICHERT J, et al. Diretrizes para cessação do tabagismo-2008. Jornal Brasileiro de Pneumologia, 2008; 34(10): 845880.

22. RICHMOND R, et al. A randomized controlled trial of a smoking cessation intervention conducted among prisoners. Addiction, 2013; 108(5): 966-974.

23. SCHNOLL RA, et al. Long-term nicotine replacement therapy: a randomized clinical trial. JAMA internal medicine, 2015; 175(4): 504-511.

24. WADGAVE U, NAGESH L. Nicotine replacement therapy: an overview. International journal of health sciences, 2016 ; 10(3): 425.

25. WORLD HEALTH ORGANIZATION. global report on trends in prevalence of tobacco smoking 2000-2025, second edition. Geneva: World Health Organization, 2018. 